\title{
Rationally connected rational double covers of primitive Fano varieties
}

\author{
Aleksandr V. Pukhlikov
}

\begin{abstract}
We show that for a Zariski general hypersurface $V$ of degree $M+1$ in $\mathbb{P}^{M+1}$ for $M \geqslant 5$ there are no Galois rational covers $X \rightarrow V$ of degree $d \geqslant 2$ with an abelian Galois group, where $X$ is a rationally connected variety. In particular, there are no rational maps $X \rightarrow V$ of degree 2 with $X$ rationally connected. This fact is true for many other families of primitive Fano varieties as well and motivates a conjecture on absolute rigidity of primitive Fano varieties.
\end{abstract}

Keywords. Fano variety; rational map; free family of rational curves; canonical singularity; branch divisor

2020 Mathematics Subject Classification. 14E05; 14E07

[Français]

Revêtements rationnels doubles rationnellement connexes des variétés de Fano primitives

Résumé. Nous montrons qu'une hypersurface $V$ de degré $M+1$ de $\mathbb{P}^{M+1}$ avec $M \geqslant 5$ n'admet aucun revêtement rationnel galoisien $X \rightarrow V$ de degré $d \geqslant 2$ et de groupe de Galois abélien avec $X$ rationnellement connexe. En particulier, $V$ n'admet aucune application rationnelle $X \rightarrow V$ de degré 2 avec $X$ rationnellement connexe. Ceci est également vérifié par beaucoup d'autres familles de variétés de Fano primitives et motive une conjecture concernant la rigidité absolue des variétés de Fano primitives.

Received by the Editors on November 3, 2019, and in final form on September 3, 2020.

Accepted on October 5, 2020.

Aleksandr V. Pukhlikov

Department of Mathematical Sciences, The University of Liverpool

e-mail:pukh@liverpool.ac.uk

The author thanks The Leverhulme Trust for the support (Research Project Grant RPG-2016-279).

(C) by the author(s)

This work is licensed under http://creativecommons.org/licenses/by-sa/4.0/ 


\section{Contents}

1. Statement of the main results . . . . . . . . . . . . . . . . . . . . . . . . . 2

2. Divisorially canonical varieties . . . . . . . . . . . . . . . . . . . . . . . . 3

3. Proof of Theorem 1.3 . . . . . . . . . . . . . . . . . . . . . . . . . . . . 4

4. Resolution of a family of curves . . . . . . . . . . . . . . . . . . . . . . . . . . . 7

5. Construction of the cyclic cover . . . . . . . . . . . . . . . . . . . . . . . . . . . . . . 9

6. Generalizations . . . . . . . . . . . . . . . . . . . . . . . . . . . . . . 11

7. Historical remarks and acknowledgements . . . . . . . . . . . . . . . . . . . 12

References. . . . . . . . . . . . . . . . . . . . . . . . . 12

\section{Statement of the main results}

One of the most challenging problems in the modern birational geometry is the unirationality problem: for a given rationally connected projective variety $V$, is there a rational dominant map $\mathbb{P}^{M} \rightarrow V$ ? While in the past 50 years a huge progress has been made in solving the rationality problem, the unirationality still remains an unknown territory. There are lots of explicit examples and constructions of unirational varieties but not a single result about non-unirationality (in the rationally connected category). During the past century (starting with Fano himself and maybe even earlier) the non-unirationality was conjectured for various classes of algebraic varieties, but the questions remained unanswered.

The aim of the present paper is to prove a theorem that implies, in particular, that there are no rational maps of degree 2

$$
\mathbb{P}^{M} \stackrel{2: 1}{\rightarrow} V
$$

for a Zariski general hypersurface $V \subset \mathbb{P}^{M+1}$ of degree $M+1$ (which is a Fano variety of index 1, in particular, a rationally connected variety). In fact, there are no rational maps of degree 2

$$
X \stackrel{2: 1}{X} V
$$

with $X$ rationally connected for such hypersurfaces $V$. We now state the main theorem.

Let $V$ be a projective factorial variety with at most terminal singularities, such that Pic $V=\mathbb{Z} K_{V}$ and the anticanonical class $\left(-K_{V}\right)$ is ample (that is, a primitive Fano variety). We work over the ground field $\mathbb{C}$ of complex numbers.

Definition 1.1 ( $c f$. [Puk05]). The Fano variety $V$, described above, is divisorially canonical if for every effective divisor $D \sim-n K_{V}, n \geqslant 1$, the pair $\left(V, \frac{1}{n} D\right)$ is canonical; that is to say, for every exceptional prime divisor $E$ over $V$ the inequality

$$
\operatorname{ord}_{E} D \leqslant n \cdot a(E),
$$

where $a(E)$ is the discrepancy of $E$ with respect to $V$, holds.

Apart from the divisorial canonicity, we will need the following technical conditions. 
$(\star 1)$ For every anticanonical divisor $R \in\left|-K_{V}\right|$, every prime number $p \geqslant 2$ and any, possibly reducible, closed subset $Y \subset V$ of codimension $\geqslant 2$ there is a non-singular curve $N \subset V$ such that

$$
p \nmid\left(N \cdot K_{V}\right) \text {, }
$$

$N \cap Y=\emptyset$ and $N$ meets $R$ transversally at non-singular points.

$(\star 2)$ For every, possibly reducible, closed subset $Y \subset V$ of codimension $\geqslant 2$ there is a non-singular rational curve $N \subset V$ such that $N \cap Y=\emptyset$.

Definition 1.2. We say that a rational dominant map $X \rightarrow Z$ of varieties of the same dimension is a rational Galois cover, if the corresponding field extension $\mathbb{C}(Z) \subset \mathbb{C}(X)$ is a Galois extension. If the corresponding Galois group is cyclic, we say that this rational map is a rational cyclic cover.

The main result of the present paper is the following claim.

Theorem 1.3. Assume that the Fano variety $V$, introduced above, is divisorially canonical and satisfies the conditions $(\star 1)$ and $(\star 2)$. Then there are no rational Galois covers $X \stackrel{d: 1}{\rightarrow} V$ with an abelian Galois group of order $d \geqslant 2$, where $X$ is a rationally connected variety.

Since the divisorial canonicity implies birational superrigidity, as an immediate consequence of Theorem 1.3, we get the following claim.

Corollary 1.4. In the assumptions of Theorem 1.3, if $X \stackrel{d: 1}{\rightarrow} V$ is a rational Galois cover with an abelian Galois group, then $d=1$ and the MMP for $X$ has the unique outcome $V$.

Since every rational map of degree 2 is a Galois rational cover with the cyclic group $C_{2}$ as the Galois group, we obtain the following claim.

Corollary 1.5. In the assumptions of Theorem 1.3, there are no rational maps $X \stackrel{2: 1}{\rightarrow} V$ of degree 2 with $X$ a rationally connected variety.

Although a particular case of Theorem 1.3, the last corollary is especially important as it covers all rational maps of degree 2 and therefore motivates the following conjecture.

Conjecture 1.6 (on absolute rigidity). If $V$ is a divisorially canonical Fano variety, then every rational dominant map $X \rightarrow V$, where $X$ is a rationally connected variety of dimension $\operatorname{dim} V$, is a birational map.

\section{Divisorially canonical varieties}

Given that the main assumption for the variety $V$ in Theorem 1.3 is divisorial canonicity, the natural question to ask now is how typical this property is in the class of Fano varieties? Let the symbol $\mathbb{P}$ stand for the complex projective space $\mathbb{P}^{M+1}$, where $M \geqslant 5$. Set $\mathcal{F}=\mathbb{P}\left(H^{0}\left(\mathbb{P}, \mathcal{O}_{\mathbb{P}}(M+1)\right)\right)$ to be the space of hypersurfaces of degree $M+1$ in $\mathbb{P}$. If a hypersurface $V \in \mathcal{F}$ is factorial, then $\operatorname{Pic} V=\mathbb{Z} H$, where $H$ is the class of a hyperplane section. In [Puk05] it was shown that a Zariski general non-singular hypersurface $V$ is divisorially canonical. Since it is not hard to check that the properties $(\star 1)$ and $(\star 2)$ are satisfied for a non-singular hypersurface $V$ (this is done below in Section 3), we obtain the following claim.

Corollary 2.1. For a Zariski general hypersurface $V \subset \mathbb{P}$ of degree $M+1$, where $M \geqslant 5$, there are no non-trivial rational Galois covers $X \stackrel{d: 1}{\rightarrow} V$ with an abelian Galois group of order $d \geqslant 2$, where $X$ is a rationally connected variety; in particular, there are no rational maps $X \rightarrow V$ of degree 2 with $X$ rationally connected

Note that in [Puk15] it was shown that for $M \geqslant 9$ there exists a Zariski open subset $\mathcal{F}_{\text {reg }} \subset \mathcal{F}$, such that every hypersurface $V \in \mathcal{F}_{\text {reg }}$ has at most quadratic singularities of rank $\geqslant 8$, so is a factorial variety with 
terminal singularities, and satisfies the property of divisorial canonicity. Moreover, for the complement $\mathcal{F} \backslash \mathcal{F}_{\text {reg }}$ the inequality

$$
\operatorname{codim}\left(\left(\mathcal{F} \backslash \mathcal{F}_{\text {reg }}\right) \subset \mathcal{F}\right) \geqslant \frac{(M-6)(M-5)}{2}-5
$$

holds. The property $(\star 1)$ is easy to show for hypersurfaces $V \in \mathcal{F}_{\text {reg }}$, see Section 3 .

Apart from Fano hypersurfaces of index 1, the divisorial canonicity was also shown for Zariski general varieties in the following families:

- double spaces of index 1 and dimension $\geqslant 3$, see [Puk05],

- a majority of the families of Fano complete intersections of index 1 in the projective space [Puk06, EP16, Puk18],

- finite, not necessarily cyclic, covers of index 1 of the projective spaces [Puk19].

This list is probably not complete: computing or estimating the $(\log )$ canonical thresholds has become a popular topic, see [CPW14, CS08, LZ19, Zhu18] and other works in this direction.

\section{Proof of Theorem 1.3}

Let us assume the converse and fix a non-trivial rational Galois cover $\sigma: X \rightarrow V$ with an abelian Galois group, where $V$ is a divisorially canonical variety, satisfying $(\star 1)$ and $(\star 2)$, and $X$ is rationally connected. Considering the field extension $\mathbb{C}(V) \subset \mathbb{C}(X)$, we can find an intermediate field which is a normal extension of $\mathbb{C}(V)$ with a cyclic group of a prime order $p \geqslant 2$ as its Galois group. Since the image of a rationally connected variety is rationally connected, we may assume that the Galois group of the original extension $\mathbb{C}(V) \subset \mathbb{C}(X)$ is a cyclic group of a prime order $p \geqslant 2$. Further, we may assume that $\sigma: X \rightarrow V$ is a morphism and $X$ is a non-singular projective variety, $\operatorname{dim} X=\operatorname{dim} V$.

We say that a family $\mathcal{L}$ of irreducible projective curves on a quasi-projective variety is free, if they sweep out a dense subset of that variety and for every subvariety $Y$ of codimension $\geqslant 2$ the subset

$$
\{L \in \mathcal{L} \mid L \cap Y \neq \emptyset\}
$$

is a proper closed subfamily of the family $\mathcal{L}$ (that is to say, a curve $L \in \mathcal{L}$ of general position does not intersect $Y$ ). Let us fix a free family $\mathcal{C}_{X}$ of non-singular rational curves on $X$.

This free family of rational curves is a crucial object in our proof. For the existence and basic properties of free families of rational curves, our reference is Kollár's book, [Kol96, Sections II.3 and IV.3]. The facts used below are well known and standard; here we explain briefly the easiest way to understand the basic geometry of such families. By Theorem 3.9 in [Kol96, Section IV.3] we have a family $\mathcal{C}_{X}$ of non-singular rational curves $C_{X}$ on $X$ such that the vector bundle $\left.T_{X}\right|_{C_{X}}$ is ample, that is, it is of the form

$$
\oplus \mathcal{O}_{\mathbb{P}^{1}}\left(\alpha_{i}\right)
$$

with all $\alpha_{i} \geqslant 1$. (This implies, in particular, that $\left(K_{X} \cdot C_{X}\right)<0$, although that inequality holds under much weaker assumptions for the family of curves.) Therefore, the infinitesimal deformations of the curve $C_{X}$ given by the sections of the vector bundle $\left.T_{X}\right|_{C_{X}}$ are unobstructed, see Points $3.3-3.5 .4$ in [Kol96, Section II.3], and if for a particular subvariety $Y \subset X$ of codimension $\geqslant 2$ and a particular curve $C_{X} \in \mathcal{C}_{X}$ the intersection $C_{X} \cap Y \neq \emptyset$, then a general deformation of $C_{X}$ in the family $\mathcal{C}_{X}$ does not meet $Y$ : we can deform the curve away from $Y$, see Proposition 3.7 in [Kol96, Section II.3].

For the same reason, for every prime divisor $\Delta \subset X$, such that $\sigma_{*}: T_{p} X \rightarrow T_{\sigma(p)} V$ is not an isomorphism for a point of general position $p \in \Delta$ (this is true, in particular, if $\operatorname{codim}(\sigma(\Delta) \subset V) \geqslant 2$ ), a general curve $C_{X} \in \mathcal{C}_{X}$ meets $\Delta$ transversally at points of general position. (There are finitely many such divisors, so the assumption that a general $C_{X}$ meets every $\Delta$ at points of general position is justified by the family $\mathcal{C}_{X}$ being 
free; the transversality follows from the ampleness of the restriction of the tangent bundle $T_{X}$ onto $C_{X}$, see the references above.)

The same deformation arguments (see the proof of Proposition 3.7 in [Kol96, Section II.3] and Theorems 1.7 and 1.8 in [Kol96, Section II.1] give us that for a general curve $C_{X} \in \mathcal{C}_{X}$ the morphism

$$
\left.\sigma\right|_{C_{X}}: C_{X} \rightarrow \sigma\left(C_{X}\right)
$$

is birational (again, this comes from the ampleness of $T_{X}$ restricted onto a general $C_{X}$, see Proposition 3.5 and Corollary 3.5.3 in [Kol96, Section II.3]: when we deform the curve $C_{X}$, any two distinct points $p \neq q$ on this curve vary independently, so that if for a particular curve $C_{X}$ the morphism $\left.\sigma\right|_{C_{X}}$ is not birational, choosing two points $p \neq q$ such that $\sigma(p)=\sigma(q)$, we can see that a general deformation of this curve satisfies the required property.)

Since in the subsequent arguments we need only the general curve $C_{X} \in \mathcal{C}_{X}$, we will remove from $\mathcal{C}_{X}$ proper closed subsets when we need it, without special comments and keeping the same notation $\mathcal{C}_{X}$. Let $\mathcal{C}_{V}=\sigma_{*} \mathcal{C}_{X}$ be the image of that family on $V$. The family $\mathcal{C}_{V}$ is, generally speaking, not free: if the $\sigma$-image of a prime divisor $\Delta \subset X$ is of codimension $\geqslant 2$, then the general curve $C_{V} \in \mathcal{C}_{V}$ meets $\sigma(\Delta)$.

Proposition 3.1. There is a birational morphism $\varphi: V^{+} \rightarrow V$, where $V^{+}$is a non-singular projective variety, such that the strict transform $\mathcal{C}_{V}^{+}$of the family $\mathcal{C}_{V}$ on $V^{+}$is a free family of curves.

Proof. It is given in Section 4. (Here of course the strict transform of a family of irreducible curves is the family of irreducible curves, a general curve in which is the strict transform of a general curve in the original family. The parameterizing space of the new family is, generally speaking, a Zariski open subset of the parameterizing space of the original family.)

Proposition 3.2. There is a non-singular quasi-projective variety $U_{X}$, a birational map $\varphi_{X}: U_{X} \rightarrow X$ and a Zariski open subset $U \subset V^{+}$, such that:

(i) the rational map

$$
\sigma_{*}=\varphi^{-1} \circ \sigma \circ \varphi_{X}: U_{X} \rightarrow V^{+}
$$

extends to a morphism $\sigma_{U}: U_{X} \rightarrow V^{+}$, the image of which is $U$,

(ii) the inequality

$$
\operatorname{codim}\left(\left(V^{+} \backslash U\right) \subset V^{+}\right) \geqslant 2
$$

holds,

(iii) the map $\sigma_{U}: U_{X} \rightarrow U$ is a cyclic cover of order $p$, branched over a non-singular hypersurface $W \subset U$.

The proof is given in Section 5 .

To make the statement of Proposition 3.2 more visual, we arrange the maps into the following commutative diagram:

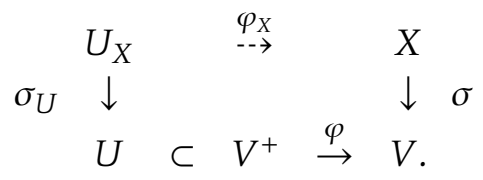

Assuming Propositions 3.1 and 3.2, let us complete the proof of Theorem 1.3. By the inequality (ii) of Proposition 3.2 a general curve $C \in \mathcal{C}_{V}^{+}$does not meet the closed set $V^{+} \backslash U$ and for that reason is contained entirely in $U$. Therefore, for some open subfamily $\mathcal{C} \subset \mathcal{C}_{V}^{+}$all curves $C \in \mathcal{C}$ are entirely contained in $U$, so that $\mathcal{C}$ is a family of irreducible projective rational curves sweeping out $U$. By the construction of the family $\mathcal{C}$ for a general curve $C \in \mathcal{C}$ its preimage

$$
\sigma_{U}^{-1}(C)=C_{1} \cup C_{2} \cup \cdots \cup C_{p}
$$


is a union of $p$ distinct rational curves on the quasi-projective variety $U_{X}$. Indeed, since for a general curve $C_{X} \in \mathcal{C}_{X}$ the morphism $\left.\sigma\right|_{C_{X}}$ is birational, and by construction of the family $\mathcal{C}$, we may assume that $C_{1}$ belongs to a family of irreducible rational curves sweeping out $U_{X}$ and the morphism $C_{1} \rightarrow C$ is birational. Then the other curves $C_{i}, i \neq 1$, are the images of $C_{1}$ under the action of elements of the cyclic Galois group; in particular, they also belong to families of irreducible rational curves sweeping out $U_{X}$, so that

$$
\left(C_{i} \cdot K_{U}\right)<0,
$$

$i=1, \ldots, p$, where $K_{U}$ is the canonical class of the variety $U_{X}$. Write

$$
K_{V}^{+}=-\varphi^{*} H+\sum_{i \in I} a_{i} E_{i}
$$

for the canonical class of the variety $V^{+}$, where $E_{i} \subset V^{+}$are all the prime $\varphi$-exceptional divisors and $a_{i}>0$ are their discrepancies with respect to $V$ and $H=-K_{V}$ is the ample anticanonical generator of Pic $V$. (The notation $K_{V}^{+}$looks better than the standard symbol $K_{V^{+}}$.) Denoting the restrictions of the divisorial classes onto $U$ by the same symbols and omitting the symbol $\varphi^{*}$, we get

$$
K_{U}=\sigma_{U}^{*}\left(K_{V}^{+}+\left(1-\frac{1}{p}\right) W\right)
$$

where $W \subset U$ is a non-singular hypersurface, over which the cyclic cover $\sigma_{U}$ is branched. Collecting separately the components of the hypersurface $W$, which are divisorial on $V$ and $\varphi$-exceptional, write

$$
W=W_{\mathrm{div}}+W_{\mathrm{exc}},
$$

where $W_{\text {div }}=n H-\sum_{i \in I} b_{i} E_{i}$ with $b_{i} \in \mathbb{Z}_{+}$and $W_{\text {exc }}=\sum_{i \in I} c_{i} E_{i}$ with $c_{i} \in\{0,1\}$. Obviously, the inequalities $\left(C \cdot K_{V}^{+}\right)<0,\left(C \cdot W_{\text {div }}\right) \geqslant 0$ and $\left(C \cdot W_{\text {exc }}\right) \geqslant 0$ hold. At the same time,

$$
\left(C_{i} \cdot K_{U}\right)=\left(C \cdot K_{V}^{+}\right)+\left(1-\frac{1}{p}\right)(C \cdot W)<0 .
$$

Assume first that $n \geqslant 2$. Adding to the left hand side of $(3.2)$ the non-positive expression $\left[\left(1-\frac{1}{p}\right) n-1\right]\left(C \cdot K_{V}^{+}\right)$, we obtain the inequality

$$
n\left(C \cdot K_{V}^{+}\right)+(C \cdot W)=\left(C \cdot \sum_{i \in I}\left(n a_{i}-b_{i}+c_{i}\right) E_{i}\right)<0,
$$

so that for some $i \in I$ we have

$$
b_{i}>n a_{i}+c_{i} \geqslant n \cdot a_{i}
$$

and the pair $\left(V, \frac{1}{n} \varphi_{*} W_{\text {div }}\right)$ with $\varphi_{*} W_{\text {div }} \sim n H$ is not canonical, which contradicts the divisorial canonicity of the variety $V$. Therefore, $n=1$ or 0 .

The case $n=1$ is impossible: in that case $\varphi_{*} W_{\text {div }}=\varphi\left(W_{\text {div }}\right) \subset V$ is an anticanonical divisor on the variety $V$ and we apply the assumption $(\star 1)$ for the prime divisor $R=\varphi\left(W_{\text {div }}\right)$ : we take a non-singular curve $N$ (of arbitrary genus) such that $p X\left(N \cdot K_{V}\right)$, which does not meet the set

$$
\varphi\left(V^{+} \backslash U\right) \cup \varphi\left(\bigcup_{i \in I} E_{i}\right)
$$

and meets the divisor $R$ transversally at points of general position. Its strict transform $N^{+}$is contained entirely in $U$, and moreover,

$$
\sigma_{U}^{-1}\left(N^{+}\right) \rightarrow N^{+}
$$

is a cyclic cover of a non-singular curve, branched over a set of $(N \cdot R)$ points, the number of which is not divisible by $p$, which is impossible. 
The case $n=0$ is also impossible. Here we apply the assumption ( $\star 2)$, taking on the variety $V$ a non-singular rational curve $N$, which does not meet the set (3.4). Then the strict transform $N^{+} \subset V^{+}$is contained entirely in $U$, so that

$$
\sigma_{U}^{-1}\left(N^{+}\right) \rightarrow N^{+}
$$

is a non-ramified $p$-cyclic cover of a non-singular rational curve, which is impossible.

Proof of Corollary 2.1. Given that Zariski general hypersurfaces $V \in \mathcal{F}$ were shown in [Puk05] to be divisorially canonical, it remains to check that $(\star 1)$ and $(\star 2)$ hold for a general hypersurface $V$. In fact, they are satisfied for any non-singular hypersurface $V \subset \mathbb{P}$ of degree $(M+1):(\star 2)$ is true because $V$ is a non-singular Fano variety, and for $(\star 1)$ we have the following simple argument. If $p \nmid \operatorname{deg} V$, then a section of $V$ by a general 2 -plane in $\mathbb{P}$ does the job. Let us assume that $p \mid \operatorname{deg} V$.

A general line $L \subset V$ meets the hyperplane section $R$ transversally at one point. However, it might happen that $L \cap Y \neq \emptyset$. In any case, since the lines on $V$ sweep out a divisor, we may assume that $L \not \subset Y$, so that $L \cap Y$ is a finite set of points.

Now take a general plane $P \subset \mathbb{P}$ containing $L$, and let $N \subset P$ be the residual curve of the intersection $P \cap V=L+N$. Making sure that $P$ is not contained in the hyperplanes in $\mathbb{P}$ that are tangent to $V$ at the points in $L \cap Y$ and taking into account that the image of $Y$ with respect to the projection from the line $L$ is a proper closed subset of $\mathbb{P}^{M-1}$, we may assume that $N \cap Y=\emptyset$. As

$$
\operatorname{deg} N=\operatorname{deg} V-1
$$

and $p \mid \operatorname{deg} V$, the curve $N$ is what we need.

Remark 3.3. The property ( $\star 1)$ is easy to show for singular hypersurfaces $V \in \mathcal{F}_{\text {reg }}$, considered in [Puk15]. Recall [Puk15, Section 3, Subsection 3.2] that the conditions defining the open set $\mathcal{F}_{\text {reg }}$, include the following bound for the singularities of $V$ : the hypersurface may have at most quadratic singularities of rank $\geqslant 8$, so that, in particular,

$$
\operatorname{codim}(\operatorname{Sing} V \subset V) \geqslant 7 \text {. }
$$

Therefore, a general 2-plane in $\mathbb{P}$ does not meet the closed set $\operatorname{Sing} V$. Furthermore, the regularity conditions at every singular point $o \in \operatorname{Sing} V$ ensure that there are finitely many lines through the point $o$ on $V$. Therefore, a general line $L \subset V$ does not meet the singular locus of $V$ and the proof of ( $\star 1)$, given above for a non-singular hypersurface $V$, works for any $V \in \mathcal{F}_{\text {reg }}$ word for word.

\section{Resolution of a family of curves}

In order to prove Proposition 3.1, let us construct a sequence of birational morphisms

$$
V_{0} \stackrel{\varphi_{1}}{\longleftarrow} V_{1} \stackrel{\varphi_{2}}{\longleftarrow} \ldots \stackrel{\varphi_{M-1}}{\longleftarrow} V_{M-1}
$$

of non-singular projective varieties, such that $V_{0}=V, \mathcal{C}_{0}=\mathcal{C}_{V}$ and for every closed subset $Y \subset V_{i}$ of dimension $\leqslant i-1$ a general curve $C_{i} \in \mathcal{C}_{i}$ of the strict transform of the family $\mathcal{C}_{V}$ on $V_{i}$ does not meet the subset $Y$.

For $i=0$ the last claim holds in a trivial way. We will explain in detail the first two steps of this construction: the morphisms $\varphi_{1}$ and $\varphi_{2}$. The general step $\varphi_{j}: V_{j} \rightarrow V_{j-1}$ is very similar to $\varphi_{2}$ and will be easy to understand when $\varphi_{2}$ is clear.

Step 1. Let us consider the family $\mathcal{C}_{0}=\mathcal{C}_{V}$ of curves, sweeping out the variety $V_{0}$. It is clear that the set of points $\Xi_{0} \subset V_{0}$, which are contained in all curves of the family $\mathcal{C}_{0}$, is finite. We blow up this finite set of points:

$$
\varphi_{1,1}: V_{0,1} \rightarrow V_{0,0}=V_{0}
$$


and look at the strict transform $\mathcal{C}_{0,1}$ of the family $\mathcal{C}_{0}$ on $V_{0,1}$. If there are no points that lie on all curves in $\mathcal{C}_{0,1}$, we stop. Otherwise, there is a finite set $\Xi_{0,1} \subset V_{0,1}$ of such points (obviously, $\Xi_{0,1}$ is contained in the exceptional divisor of $\left.\varphi_{1,1}\right)$ and we blow it up. Repeating, if necessary, we get a finite sequence of blow ups of finite sets of points,

$$
V_{0}=V_{0,0} \stackrel{\varphi_{1,1}}{\longleftarrow} V_{0,1} \stackrel{\varphi_{1,2}}{\longleftarrow} \ldots \stackrel{\varphi_{1, e(1)}}{\longleftarrow} V_{0, e(1)},
$$

such that for every point $p \in V_{0, e(1)}$ the curves $C_{0, e(1)} \in \mathcal{C}_{0, e(1)}$ (the last symbol means the strict transform of the family $\mathcal{C}_{0}$ on $V_{0, e(1)}$ ), containing the point $p$, form a proper closed subset of the family $\mathcal{C}_{0, e(1)}$. (That our procedure can not be infinite and must terminate, follows from considering some formal parameterizations of all branches of a general curve $C_{0} \in \mathcal{C}_{0}$ at the points of the set $\Xi_{0}$.) Set $V_{1}=V_{0, e(1)}$ and

$$
\varphi_{1}=\varphi_{1,1} \circ \cdots \circ \varphi_{1, e(1)}: V_{1} \rightarrow V_{0} .
$$

Step 2. For the next step of our construction, consider the set of curves $\Xi_{1} \subset V_{1}$, which intersect all curves of the family $\mathcal{C}_{1}$. This is a finite set of curves. Indeed, embedding the quasi-projective variety, parameterizing the curves of the family $\mathcal{C}_{1}$, in some projective space, and intersecting with the appropriate number of general hyperplanes, we obtain a sufficiently mobile family of curves in that quasi-projective variety, which gives us a family of surfaces $\mathcal{S}_{1}$ on $V_{1}$, such every surface $S_{1} \in \mathcal{S}_{1}$ contains all curves of the set $\Xi_{1}$. Now it is clear that the set $\Xi_{1}$ is finite.

If $D_{1}$ is a general divisor of a very ample system on $V_{1}$, then, intersecting the surfaces $S_{1} \in \mathcal{S}_{1}$ with $D_{1}$, we obtain a family of curves on $D_{1}$, each of which contains the points of the set $\Xi_{1} \cap D_{1}$. Now, arguing as at the previous step, for the family of curves $\left(S_{1} \cap D_{1}, S_{1} \in \mathcal{S}_{1}\right)$, we see that there is a finite sequence of birational morphisms

$$
V_{1}=V_{1,0} \stackrel{\varphi_{2,1}}{\longleftarrow} V_{1,1} \stackrel{\varphi_{2,2}}{\longleftarrow} \ldots \stackrel{\varphi_{2, e(2)}}{\longleftarrow} V_{1, e(2)},
$$

where $\varphi_{2,1}$ is a composition of two birational maps:

- a desingularization of the (reducible) 1-dimensional subset $\Xi_{1}$, that is, a composition of finitely many blow ups of points, transforming $\Xi_{1}$ into a disjoint union of non-singular curves, and

- the blow up of the non-singular strict transform of $\Xi_{1}$,

and, similarly, $\varphi_{2, i}$ first resolves the singularities of the 1-dimensional set $\Xi_{1, i-1} \subset V_{1, i-1}$, which is the union of all irreducible curves on $V_{1, i-1}$, intersecting all curves of the family $\mathcal{C}_{1, i-1}$ (the strict transform of the family $\mathcal{C}_{1}$ on $\left.V_{1, i-1}\right)$, and then blows up the non-singular strict transform of the reducible curve $\Xi_{1, i-1}$. Note that by construction the image of every curve in $\Xi_{1, i-1}$ on $V_{1}$ is one of the curves of the set $\Xi_{1}$ (otherwise, all curves of the family $\mathcal{C}_{1}$ would have passed through some point, which is not true). Our procedure terminates for the same reason as at Step 1 (looking at the family of curves $\left(S_{1} \cap D_{1}, S_{1} \in \mathcal{S}_{1}\right)$ that was used above). Finally, on $V_{1, e(2)}$ there are no curves meeting all curves of the family $\mathcal{C}_{1, e(2)}$. We set $V_{2}=V_{1, e(2)}, \mathcal{C}_{2}=\mathcal{C}_{1, e(2)}$ and

$$
\varphi_{2}=\varphi_{2,1} \circ \cdots \circ \varphi_{2, e(2)}: V_{2} \rightarrow V_{1} .
$$

Carrying on in the same spirit, we construct the whole sequence (4.1). Let us consider briefly the general step of our construction.

Step $j$. By the previous arguments, if an irreducible subvariety intersects all curves of the family $\mathcal{C}_{j-1}$ on $V_{j-1}$, it has dimension $\geqslant j-1$. If there are no irreducible subvarieties of dimension $j-1$, intersecting all curves in $\mathcal{C}_{j-1}$, then there is nothing to do: we set $V_{j}=V_{j-1}$ and $\varphi_{j}$ is the identity morphism. Otherwise, let $\Xi_{j-1} \subset V_{j-1}$ be the union of all irreducible subvarieties of dimension $j-1$ meeting all curves in $\mathcal{C}_{j-1}$. Embedding the quasi-projective variety, parameterizing the curves of the family $\mathcal{C}_{j-1}$ in a projective space and intersecting it with the appropriate number of general hyperplanes, we obtain a sufficiently mobile family of subvarieties of dimension $j-1$ in that quasi-projective variety, which gives us a family $\mathcal{S}_{j-1}$ of 
irreducible subvarieties of dimension $j$ on $V_{j-1}$, such that every subvariety $S_{j-1} \in \mathcal{S}_{j-1}$ contains $\Xi_{j-1}$; in particular, $\Xi_{j-1}$ is a Zariski closed subset, and hence it contains finitely many irreducible components. If

$$
D_{1}, \quad \ldots, \quad D_{j-1}
$$

is a general tuple of divisors in a very ample linear system on $V_{j-1}$, then, intersecting the subvarieties $S_{j-1} \in \mathcal{S}_{j-1}$ with $D_{1} \cap \cdots \cap D_{j-1}$, we obtain a family of curves on $D_{1} \cap \cdots \cap D_{j-1}$, each of which contains the points of the set $\Xi_{j-1} \cap D_{1} \cap \cdots \cap D_{j-1}$. Now, arguing as at Step 2, we see that there is a finite sequence of birational morphisms

$$
V_{j-1}=V_{j-1,0} \stackrel{\varphi_{j, 1}}{\longleftarrow} V_{j-1,1} \stackrel{\varphi_{j, 2}}{\longleftarrow} \ldots \stackrel{\varphi_{j, e(j)}}{\longleftarrow} V_{j-1, e(j)},
$$

where $\varphi_{j, 1}$ is a composition of two birational maps:

- a desingularization of the (reducible) $(j-1)$-dimensional closed subset $\Xi_{j-1}$, that is, a composition of finitely many blow ups of subvarieties of dimension $\leqslant j-2$, transforming $\Xi_{j-1}$ into a disjoint union of non-singular subvarieties of dimension $j-1$, and

- the blow up of the non-singular strict transform of $\Xi_{j-1}$.

Similarly, $\varphi_{j, i}$ first resolves the singularities of the closed set $\Xi_{j-1, i-1} \subset V_{j-1, i-1}$, which is the union of all irreducible subvarieties on $V_{j-1, i-1}$, intersecting all curves of the family $\mathcal{C}_{j-1, i-1}$ (the strict transform of the family $\mathcal{C}_{j-1}$ on $\left.V_{j-1, i-1}\right)$, and then blows up the non-singular strict transform of the closed set $\Xi_{j-1, i-1}$. Again, as at Step 2, the image of every component of the closed set $\Xi_{j-1, i-1}$ on $V_{j-1}$ is one of the components of the set $\Xi_{j-1}$ - otherwise, every curve in $\mathcal{C}_{j-1}$ would have met some irreducible subvariety of dimension $\leqslant j-2$, which is not true by the previous steps of our construction. Our procedure terminates for the same reason as at Steps 1 and 2 (using the family of curves

$$
\left(S_{j-1} \cap D_{1} \cap \cdots \cap D_{j-1}, S_{j-1} \in \mathcal{S}_{j-1}\right)
$$

on $\left.D_{1} \cap \cdots \cap D_{j-1}\right)$. Finally, on $V_{j-1, e(j)}$ there are no subvarieties of dimension $\leq j-1$, meeting all curves of the family $\mathcal{C}_{j-1, e(j)}$. We set $V_{j}=V_{j-1, e(j)}, \mathcal{C}_{j}=\mathcal{C}_{j-1, e(j)}$ and

$$
\varphi_{j}=\varphi_{j, 1} \circ \cdots \circ \varphi_{j, e(j)}: V_{j} \rightarrow V_{j-1} .
$$

Having constructed the sequence (4.1), set $V^{+}=V_{M-1}$ and

$$
\varphi=\varphi_{0} \circ \varphi_{1} \circ \cdots \circ \varphi_{M-1}: V^{+} \rightarrow V .
$$

Obviously, $V^{+}$has the property, described in the statement of the proposition.

\section{Construction of the cyclic cover}

Let us show Proposition 3.2. The field extension $\mathbb{C}(V) \subset \mathbb{C}(X)$ is generated by some element $\xi \in \mathbb{C}(X)$, satisfying the equation

$$
\xi^{p}-q=0
$$

for some rational function $q \in \mathbb{C}(V)=\mathbb{C}\left(V^{+}\right)$. For some effective divisor $R$ on $V^{+}$and sections $a_{0}, a_{1} \in$ $\mathcal{O}_{V^{+}}(R)$ we can write $q=a_{0} / a_{1}$. Consider the hypersurface

$$
\{G=0\} \subset V^{+} \times \mathbb{P}_{\left(x_{0}: x_{1}\right)}^{1},
$$

where

$$
G=a_{1} x_{1}^{p}-a_{0} x_{0}^{p} \in H^{0}\left(V^{+} \times \mathbb{P}^{1}, \operatorname{pr}_{V}^{*} \mathcal{O}_{V^{+}}(R) \otimes \operatorname{pr}_{\mathbb{P}}^{*} \mathcal{O}_{\mathbb{P}^{1}}(p)\right),
$$

the symbols $\operatorname{pr}_{V}$ and $\operatorname{pr}_{\mathbb{P}}$ mean the projections of $V^{+} \times \mathbb{P}^{1}$ onto the first and second factor, respectively. This hypersurface is, generally speaking, reducible, but has a unique irreducible component $X_{0}$, such that $\operatorname{pr}_{V}\left(X_{0}\right)=V^{+}$. 
If for some prime divisor $T \subset V^{+}$the hypersurface $\{G=0\}$ contains $\operatorname{pr}_{V}^{-1}(T)$, then $a_{0},\left.a_{1}\right|_{T} \equiv 0$, so that, replacing $R$ by $R-m T$, where

and $a_{0}, a_{1} \in \mathcal{O}_{V^{+}}(R)$ by

$$
m=\min \left\{\operatorname{ord}_{T}\left(a_{0}\right), \operatorname{ord}_{T}\left(a_{1}\right)\right\}
$$

$$
\frac{a_{0}}{s_{T}^{m}}, \frac{a_{1}}{s_{T}^{m}} \in \mathcal{O}_{V^{+}}(R-m T),
$$

where $s_{T} \in \mathcal{O}_{V^{+}}(T)$ is the section, corresponding to the divisor $T$, we remove the component $\operatorname{pr}_{V}^{-1}(T)$. Therefore, we may assume from the beginning that the sections $a_{0}, a_{1} \in \mathcal{O}_{V^{+}}(R)$ do not vanish simultaneously on any prime divisor $T \subset V^{+}$and $\{G=0\}=X_{0} \subset V^{+} \times \mathbb{P}^{1}$ is an irreducible hypersurface, by construction birational to the original variety $X$.

Now let us consider the singularities of the variety $X_{0}$. Assume that for some prime divisor $T \subset V^{+}$there is a subvariety $T_{X} \subset \operatorname{Sing} X_{0}$, such that

$$
\operatorname{pr}_{V}\left(T_{X}\right)=T \text {. }
$$

Let $\mathcal{T}$ be the set of all prime divisors on $V^{+}$with that property. By what was said above, we may assume that say, $\left.a_{1}\right|_{T} \not \equiv 0$, which implies that

$$
\operatorname{ord}_{T} a_{0} \geqslant 2 \text {. }
$$

Removing the set of common zeros of the sections $a_{0}, a_{1} \in \mathcal{O}_{V^{+}}(R)$, the pairwise intersections of the divisors $T \in \mathcal{T}$ (if $\sharp \mathcal{T} \geqslant 2$ ) and the sets of singular points Sing $T$ for all $T \in \mathcal{T}$, we obtain a Zariski open set $U \subset V^{+}$, such that

- $\operatorname{codim}\left(\left(V^{+} \backslash U\right) \subset V^{+}\right) \geqslant 2$,

- the morphism $X_{0} \cap \operatorname{pr}_{V}^{-1}(U) \rightarrow U$ is a finite morphism of degree $\mathrm{p}$ and

- for every divisor $T \in \mathcal{T}$ the quasi-projective varieties $T \cap U$ and $T_{X} \cap \operatorname{pr}_{V}^{-1}(U)$ are non-singular, and moreover, the projection $\operatorname{pr}_{V}$ gives an isomorphism of these varieties, which we for simplicity of notations write down again as $T$ and $T_{X}$.

Now distinct varieties in $\mathcal{T}$ are disjoint.

Furthermore, we may assume that for every $T \in \mathcal{T}$ the section $a_{i}, i=0,1$, which does not vanish identically on $T$, is everywhere non-zero on $T \cap U$, so that if say $\left.a_{1}\right|_{T} \not \equiv 0$, then the hypersurface $X_{0} \cap \operatorname{pr}_{V}^{-1}(U)$ over a neighborhood of the divisor $T \cap U$ is contained in the open subset

$$
\left\{x_{0} \neq 0\right\}=U \times \mathbb{A}_{z}^{1},
$$

with $z=x_{1} / x_{0}$ and given by the equation

$$
a_{1} z^{p}-a_{0}=0,
$$

so that the corresponding subvariety $T_{X} \subset \operatorname{Sing} X_{0}$ over $T \cap U$ is given in $T \times \mathbb{A}_{z}^{1}$ by the equation $z=0$.

Thus we have constructed a locally trivial $\mathbb{P}^{1}$-bundle $\mathcal{X}_{1}$ over a non-singular quasi-projective variety $U$ (of course, $\mathcal{X}_{1}=U \times \mathbb{P}^{1}$ ) and a hypersurface $X_{1}=X_{0} \cap \operatorname{pr}_{V}^{-1}(U) \subset \mathcal{X}_{1}$ which is a cyclic cover of the prime order $p$ over $U$. The projection $\mathcal{X}_{1} \rightarrow U$ will be denoted by the symbol $\pi_{1}$. The cyclic cover $X_{1} \rightarrow U$ has all properties required in Proposition 3.2 except for $X_{1}$ being non-singular. The rest of our proof of the proposition is removing the singularities of $X_{1}$. This has to be done carefully, preserving the cyclic cover.

We desingularize the covering variety in two steps. The first step is constructing a sequence of locally trivial $\mathbb{P}^{1}$-bundles over $U$ :

$$
\mathcal{X}_{1} \stackrel{\beta_{1}}{\longleftarrow} \mathcal{X}_{2} \stackrel{\beta_{2}}{\longleftarrow} \ldots \stackrel{\beta_{k-1}}{\longleftarrow} \mathcal{X}_{k},
$$

where $\beta_{i}$ is an elementary birational transformation over $U$,

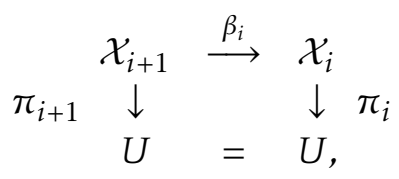


defined in the following way. Assume that the strict transform $X_{i} \subset \mathcal{X}_{i}$ of the hypersurface $X_{1}$ is singular along a subvariety $\pi_{i}^{-1}(T) \cap X_{i}=T_{i}$ for some $T \in \mathcal{T}$. With respect to a certain trivialization of the $\mathbb{P}^{1}$-bundle $\mathcal{X}_{i} / U$ over an open subset, intersecting the divisor $T$, the hypersurface $X_{i}$ is given by the equation

$$
a_{i, 1} x_{1}^{p}-a_{i, 0} x_{0}^{p}=0 \text {, }
$$

where, say, $\left.a_{i, 1}\right|_{T} \not \equiv 0$ and ord $a_{T} a_{i, 0} \geqslant 2$. Assume, furthermore, that in fact the inequality

$$
\operatorname{ord}_{T} a_{i, 0} \geqslant p
$$

holds. Then the birational transformation $\beta_{i}: \mathcal{X}_{i+1} \rightarrow \mathcal{X}_{i}$ is the composition of the blow up of the subvariety $T_{i}$ (by construction, this non-singular subvariety is a section of the $\mathbb{P}^{1}$-bundle $\pi_{i}^{-1}(T) \rightarrow T$ ) and subsequent contraction of the strict transform of the hypersurface $\pi_{i}^{-1}(T)$. Elementary computations with local parameters, using the explicit presentation described above, show that locally in a neighborhood of the generic point of the divisor $T$ the hypersurface $X_{i+1}$ is given by the equation

$$
a_{i+1,1} x_{1}^{p}-a_{i+1,0} x_{0}^{p}=0,
$$

where $\left.a_{i+1,1}\right|_{T} \not \equiv 0$ and $\operatorname{ord}_{T} a_{i+1,0}=\operatorname{ord}_{T} a_{i, 0}-p$. Therefore, after finitely many elementary transformations of the ambient $\mathbb{P}^{1}$-bundle we obtain a locally trivial $\mathbb{P}^{1}$-bundle

$$
\pi_{k}: \mathcal{X}_{k} \rightarrow U
$$

such that if the strict transform $X_{k} \subset \mathcal{X}_{k}$ of the hypersurface $X_{1}$ is singular along a subvariety $\pi_{k}^{-1}(T) \cap X_{k}=\widetilde{T}$ for a $T \in \mathcal{T}$, then over the general point of $T$ the hypersurface $X_{k} \subset \mathcal{X}_{k}$ is given by the equation

$$
a_{T, 1} x_{1}^{p}-a_{T, 0} x_{0}^{p}=0,
$$

where, say where, say, $a_{T, 1}$ does not vanish on $T$, and

$$
\operatorname{ord}_{T} a_{T, 0}=l_{T} \in\{2, \ldots, p-1\} \text {. }
$$

This means that if $p=2$, then the proof of Proposition 3.2 is completed: the variety $X_{k}$ is a non-singular cyclic cover of $U$.

Assume now that $p \geqslant 3$. Then along the subvariety $\pi_{k}^{-1}(T) \cap X_{k}$ the variety $X_{k}$ has a cuspidal singularity of the type

$$
t^{p}-s^{l_{T}}=0
$$

Taking the normalization of the variety $X_{k}$ or applying the obvious sequence of blow ups, we obtain the required variety $U_{X}$ that covers $U$ cyclically, and complete the proof of Proposition 3.2 for a cyclic cover of degree $p \geqslant 3$. This completes the proof of Theorem 1.3.

\section{Generalizations}

First of all, given that the divisorial canonicity has been shown in [Puk06, EP16, Puk18] for many families of Fano complete intersections of index 1 in the projective space, we obtain the following generalization of Corollary 2.1.

Corollary 6.1. For a divisorially canonical non-singular Fano complete intersection $V \subset \mathbb{P}^{M+k}$ of codimension $k$ and index 1, there are no non-trivial rational Galois covers $X \stackrel{d: 1}{\rightarrow} V$ with an abelian Galois group of order $d \geqslant 2$, where $X$ is a rationally connected variety; in particular, there are no rational maps $X \rightarrow V$ of degree 2 with $X$ rationally connected.

Proof. We need only to check that the properties $(\star 1)$ and $(\star 2)$ are satisfied for a non-singular complete intersection $V$. The property $(\star 2)$ is automatic and in order to show $(\star 1)$, we argue as in the proof of 
Corollary 2.1: if $p \nmid \operatorname{deg} V$, then a section of $V$ by a general linear $(k+1)$-subspace in $\mathbb{P}^{M+k}$ works as $N$, and if $p \mid \operatorname{deg} V$, we obtain $N$ as the residual curve,

$$
P \cap V=L+N,
$$

where $L \subset V$ is a general line and $P$ a general linear $(k+1)$-subspace in $\mathbb{P}^{M+k}$, containing $L$.

Remark 6.2. It seems that the claim of Theorem 1.3 is true for rationally connected Galois rational covers with an arbitrary Galois group, not necessarily an abelian one. We may assume that $X$ is a non-singular projective variety and the Galois group of the extension $\mathbb{C}(V) \subset \mathbb{C}(X)$ is a finite subgroup of the group Aut $X$. Now the ramification divisor of the finite cover $U_{X} \rightarrow U$ is invariant with respect to the action of the Galois group and for that reason is pulled back from $U$, so that the proof of Theorem 1.3 must work in the non-abelian case, too.

Conjecture 6.3. The claim of Theorem 1.3 holds without the assumption that the Galois group is abelian.

Remark 6.4. The fact that there are no rationally connected rational double covers for a hypersurface $V \subset \mathbb{P}$ of degree $M+1$, where $M \geqslant 5$, was obtained from the property that every pair $\left(V, \frac{1}{n} D\right)$, where $D \sim n H$, is canonical. However, for certain special hypersurfaces $V$ this is not true: for instance, if $V \cap T_{o} V$ is a cone with the vertex at the point $o$. On the other hand, it seems probable that the claim of Theorem 1.3 is true for any smooth hypersurface of index 1 for $M \geqslant 5-$ and, possibly, for four-dimensional quintics and three-dimensional quartics, for which the technique of [Puk05] does not prove the property of divisorial canonicity. Note also that it is hard to see any obstructions for the Conjecture 1.6 to be true at least for any smooth hypersurface of index 1 and dimension $\geqslant 4$. At the same time, as Segre's example shows, for certain smooth three-dimensional quartics Conjecture 1.6 does not hold because they are unirational, see [IM71].

\section{Historical remarks and acknowledgements}

As far as I know, the first time when a conjecture on non-unirationality of certain rationally connected three-folds (in that case, conic bundles) was explicitly stated, was in [Fan31] by Gino Fano himself. In [Kol98] Kollár suggested an approach to proving the non-unirationality of primitive Fano hypersurfaces: if the variety parameterizing rational curves of some arbitrary fixed degree passing through a general point $o \in V$ contains no rational curves, then there are no rational surfaces on $V$ containing this point, which of course implies non-unirationality. The suggested approach motivated a far reaching investigation of the space of rational curves on hypersurfaces in [BS08, BK13]. For the various explicit results on unirationality (solving the unirationality problem affirmatively) see, for instance, [HMP98, CM98, Con01, CMM09, CM06-08]. A topic that can be linked to the conjecture on absolute rigidity is endomorphisms, especially rational endomorphisms of rationally connected varieties; there are quite a few papers on that subject, see, for instance, [AKP08, HN11, ABR11, Zha12, Zha14].

The author is grateful to the colleagues in the Divisions of Algebraic Geometry and Algebra at Steklov Institute of Mathematics for the interest to his work, and to the colleagues-algebraic geometers at the University of Liverpool for the general support.

Finally, I would like to thank both referees for their work on my paper and a number of useful suggestions.

\section{References}

[ABR11] E. Amerik, F. Bogomolov and M. Rovinsky, Remarks on endomorphisms and rational points, Compos. Math. 147 (2011) 1819-1842.

[AKP08] M. Aprodu, S. Kebekus and T. Peternell, Galois coverings and endomorphisms of projective varieties, Math. Z. 260 (2008), no. 2, 431-449. 
[BK13] R. Beheshti and N. M. Kumar, Spaces of rational curves on complete intersections, Compos. Math. 149 (2013), no. 6, 1041-1060.

[BS08] R. Beheshti and J. M. Starr, Rational surfaces in index-one Fano hypersurfaces, J. Algebraic Geom. 17 (2008), no. 2, 255-274.

[Con01] A. Conte, The unirationality of all conic bundles implies the unirationality of the quartic threefold with an appendix by M. Marchisio. Lecture Notes in Pure and Appl. Math., 217, Geometric and combinatorial aspects of commutative algebra (Messina, 1999), 125-129, Dekker, New York, 2001.

[CMM09] A. Conte, M. Marchisio and J. P. Murre, On the unirationality of the quintic hypersurface containing a 3-dimensional linear space, Atti Accad. Sci. Torino Cl. Sci. Fis. Mat. Natur. 142 (2008), 89-96 (2009).

[CM98] A. Conte and J. P. Murre, On a theorem of Morin on the unirationality of the quartic fivefold, Atti Accad. Sci. Torino Cl. Sci. Fis. Mat. Natur. 132 (1998), 49-59.

[CM06-08] A. Conte and M. Marchisio, Some questions of (uni)rationality, I, II,III, Atti Accad. Sci. Torino Cl. Sci. Fis. Mat. Natur. 140 (2006), 3-6 (2007); 140 (2006), 19-20 (2007); 141 (2007), 19-21 (2008).

[CS08] I. A. Cheltsov and K. A. Shramov, Log canonical thresholds of non-singular Fano threefolds, Russian Math. Surveys 63 (2008), no. 5, 859-958.

[CPW14] I. A. Cheltsov, J. Park and J. Won, Log canonical thresholds of certain Fano hypersurfaces, Math. Z. 276 (2014), no. 1-2, 51-79.

[EP16] T. Eckl and A. V. Pukhlikov, On the global log canonical threshold of Fano complete intersections, Eur. J. Math. 2 (2016), no. 1, 291-303.

[Fan31] G. Fano, Sulle varietà algebriche a tre dimensioni aventi tutti i generi nulli, Atti del Congresso Internazionale dei Matematici, Bologna, 3-10 Settembre 1928, Zanichelli Bologna (1931) 115-121.

[HMP98] J. Harris, B. Mazur and R. Pandharipande, Hypersurfaces of low degree, Duke Math. J. 95 (1998), no. $1,125-160$.

[HN11] J.-M. Hwang and N. Nakayama, On endomorphisms of Fano manifolds of Picard number one, Pure Appl. Math. Q. 7 (2011), no. 4, Special Issue: In memory of Eckart Viehweg, 1407-1426.

[IM71] V. A. Iskovskikh and Y. I. Manin, Three-dimensional quartics and counterexamples to the Lüroth problem, Math. USSR-Sb. 15 (1971), no. 1, 141-166.

[Kol96] J. Kollár, Rational curves on algebraic varieties, Springer-Verlag, Berlin, 1996.

[Kol98] J. Kollár, Low degree polynomial equations: arithmetic, geometry and topology, European Congress of Mathematics, Vol. I (Budapest, 1996), 255-288, Progr. Math., 168, Birkhäuser, Basel, 1998.

[LZ19] Y. Liu and Z. Zhuang, Birational superrigidity and K-stability of singular Fano complete intersections, Int. Math. Res. Notices (2019), https://doi.org/10.1093/imrn/rnz148.

[Puk05] A. V. Pukhlikov, Birational geometry of Fano direct products, Izv. Math. 69 (2005), no. 6, 1225-1255.

[Puk06] _ Birational geometry of algebraic varieties with a pencil of Fano complete intersections, Manuscripta Math. 121 (2006), 491-526.

[Puk15] _ Birationally rigid Fano fibrations. II, Izv. Math. 79 (2015), no. 4, 809-837.

[Puk18] _ Canonical and log canonical thresholds of Fano complete intersections, Eur. J. Math. 4 (2018), no. 1, 381-398.

[Puk19] _ _ Canonical and log canonical thresholds of multiple projective spaces, Eur. J. Math. (2019), https://doi.org/10.1007/s40879-019-00388-7. 
[Zha12] D.-Q. Zhang, Rationality of rationally connected threefolds admitting non-isomorphic endomorphisms, Trans. Amer. Math. Soc. 364 (2012), no. 12, 6315-6333.

[Zha14] _ Invariant hypersurfaces of endomorphisms of projective varieties, Adv. Math. 252 (2014), 185203.

[Zhu18] Z. Zhuang, Birational superrigidity and K-stability of Fano complete intersections of index one, Duke Math. J. 169 (2020), no. 12, 2205-2229. 